\title{
Delirium prevalence, incidence, and implications for screening in specialist palliative care inpatient settings: A systematic review
}

\author{
Annmarie Hosie School of Nursing, The University of Notre Dame, Darlinghurst Campus, Sydney, NSW, \\ Australia; Department of Palliative Care, Calvary Health Care Sydney, Kogarah, NSW, Australia

\begin{abstract}
Patricia M Davidson School of Nursing, Midwifery and Health, University of Technology, Sydney, NSW, Australia; ImPaCCT: Improving Palliative Care through Clinical Trials (New South Wales Palliative Care Clinical Trials Group), Sydney, NSW, Australia
\end{abstract}

\begin{abstract}
Meera Agar ImPaCCT: Improving Palliative Care through Clinical Trials (New South Wales Palliative Care Clinical Trials Group), Sydney, NSW, Australia; Palliative and Supportive Services, Flinders University, Adelaide, SA, Australia; South West Sydney Clinical School, University of New South Wales, Liverpool, NSW, Australia; HammondCare, Department of Palliative Care, Braeside Hospital, Sydney, NSW, Australia
\end{abstract}

Christine R Sanderson Department of Palliative Care, Calvary Health Care Sydney, Kogarah, NSW, Australia; ImPaCCT: Improving Palliative Care through Clinical Trials (New South Wales Palliative Care Clinical Trials Group), Sydney, NSW, Australia; Palliative and Supportive Services, Flinders University, Adelaide, SA, Australia

Jane Phillips School of Nursing, The University of Notre Dame, Darlinghurst Campus, Sydney, NSW, Australia; ImPaCCT: Improving Palliative Care through Clinical Trials (New South Wales Palliative Care Clinical Trials Group), Sydney, NSW, Australia; St Vincent's Mater Health, Cunningham Centre for Palliative Care, Sydney, NSW, Australia

\begin{abstract}
Background: Delirium is a serious neuropsychiatric syndrome frequently experienced by palliative care inpatients. This syndrome is under-recognized by clinicians. While screening increases recognition, it is not a routine practice.

Aim and design: This systematic review aims to examine methods, quality, and results of delirium prevalence and incidence studies in palliative care inpatient populations and discuss implications for delirium screening.

Data sources: A systematic search of the literature identified prospective studies reporting on delirium prevalence and/or incidence in inpatient palliative care adult populations from 1980 to 2012. Papers not in English or those reporting the occurrence of symptoms not specifically identified as delirium were excluded.

Results: Of the eight included studies, the majority (98.9\%) involved participants (1079) with advanced cancer. Eight different screening and assessment tools were used. Delirium incidence ranged from $3 \%$ to $45 \%$, while delirium prevalence varied, with a range of: $13.3 \%-42.3 \%$ at admission, $26 \%-62 \%$ during admission, and increasing to $58.8 \%-88 \%$ in the weeks or hours preceding death. Studies that used the Diagnostic and Statistical Manual-Fourth Edition reported higher prevalence (42\%-88\%) and incidence (40.2\%$45 \%)$, while incidence rates were higher in studies that screened participants at least daily $(32.8 \%-45 \%)$. Hypoactive delirium was the most prevalent delirium subtype $(68 \%-86 \%$ of cases).

Conclusion: The prevalence and incidence of delirium in palliative care inpatient settings supports the need for screening. However, there is limited consensus on assessment measures or knowledge of implications of delirium screening for inpatients and families. Further research is required to develop standardized methods of delirium screening, assessment, and management that are acceptable to inpatients and families.
\end{abstract}




\section{Introduction}

Delirium is a serious neuropsychiatric syndrome in hospitalized patients, including those within palliative care settings, ${ }^{1-3}$ and is associated with increased mortality. ${ }^{4,5}$ Delirium impacts upon the patient's ability to communicate, their decision-making capacity, functional ability, and quality of life. ${ }^{1}$ Patients who recover from an episode of delirium usually recall the experience ${ }^{6,7}$ and report feeling frightened and humiliated. ${ }^{8}$ In the last days or hours of life, hyperactive delirium symptoms - commonly referred to as "terminal agitation" or "terminal restlessness" - cause distress for family members. ${ }^{9-11}$

Core symptoms for a Diagnostic and Statistical Manual (DSM), Fourth Edition (DSM-IV) diagnosis of delirium include disturbed consciousness, with reduced ability to focus, sustain, or shift attention; altered cognition or a perceptual disturbance, acute onset and fluctuating symptoms, which can be mild and fleeting or severe and persistent; and evidence of an etiological cause. ${ }^{12}$ Level of consciousness identifies the three delirium subtypes: hyperactive, hypoactive, or mixed. ${ }^{13}$ Lethargy, mood changes, and altered sleep-wake cycle can also occur, although are not required to establish a diagnosis. ${ }^{12}$

Despite numerous interventions for delirium reversal, management and support of palliative care patients with delirium being available, evidence of their effectiveness is evolving and requires further development. ${ }^{14-16}$ Identifying delirium is an important priority as approximately half of all delirium episodes are potentially reversible.4,17 Iatrogenic causes, such as opioids and benzodiazepines, underscore the importance of recognition to modify palliative care interventions. ${ }^{18,19}$ Optimal recognition and assessment of delirium is of clinical and ethical concern since sedation is commonly used to manage symptoms of restlessness and agitation in the terminal stage. ${ }^{20,21}$ Underrecognition of delirium results in interventions being inconsistently applied in palliative care..$^{22-24}$

Screening improves clinician recognition of delirium, ${ }^{25}$ yet is not routinely conducted in the inpatient palliative care setting. ${ }^{26}$ Previous reviews of delirium in palliative care settings have provided comprehensive examinations of the literature including delirium prevalence and assessment methods, ${ }^{1,3,27}$ but to date, no reviews have examined in detail the methodological quality of delirium epidemiological studies conducted in palliative care inpatient settings, nor discussed implications of results in conjunction with other evidence required to justify implementation of routine delirium screening. ${ }^{28,29}$

\section{Method}

\section{Aims and review processes}

This systematic review aims to: (1) examine prevalence and incidence of delirium and delirium subtypes in specialist palliative care inpatient settings, at various stages of patients' admission, (2) describe how delirium cases were identified and established in included studies, and (3) discuss results in relation to implementation of routine delirium screening in specialist palliative care inpatient units.

Although a meta-analysis of data was not undertaken, the Meta-Analysis of Observational Studies in Epidemiology (MOOSE) ${ }^{30}$ guidelines were followed to facilitate systematic processes in the completion and reporting of the review, where relevant.

\section{Search method}

A systematic review was undertaken between 1 December 2011 and 29 February 2012 and was limited to the studies published since 1980, when delirium was first identified within the DSM, Third Edition (DSM-III), ${ }^{31}$ up until early 2012. Prospective search questions guided the search strategy using the following search Medical Subject Headings (MeSH) and key words, along with their associated derivatives: "delirium" OR "confusion" OR "terminal agitation" OR "terminal restlessness" OR "psychomotor agitation" OR "cognitive failure" OR "disorientation" AND "palliative care" OR "death" OR "dying" OR "terminal care" OR "hospice care" OR "terminally ill” OR "end of life" AND "prevalence" OR “incidence" OR "epidemiology". Search engines used were Scopus, CINAHL, and Medline. In addition, the search terms "delirium" AND "prevalence" OR "incidence" OR "epidemiology" were employed in PubMed using the palliative care filter from CareSearch. ${ }^{32}$ Reference lists of included studies and relevant reviews ${ }^{1,3}$ were also examined to search for other potentially eligible papers.

\section{Study selection}

Criteria for inclusion of papers were prospective assessment studies reporting prevalence, incidence, or rate of occurrence of delirium, conducted within specialist palliative care inpatient settings (defined as palliative care inpatient units or hospices) with adult participants. Studies were excluded if they were not published in English, or reported the rate of occurrence of symptoms or phenomena that were not specifically categorized as delirium, such as "cognitive failure," "confusion," or "terminal agitation," as the interchangeable use of such terms has previously contributed to a lack of clarity in reporting and collating of delirium occurrence in palliative care populations. ${ }^{3}$ Two authors (A.H. and J.P.) examined the titles and abstracts of all papers to determine if they met the inclusion criteria, one author (A.H.) extracted the data from potentially relevant studies $(n=13)$ and this guided decision making (A.H. and J.P.) about inclusion of studies. 


\section{Assessment of methodological quality of included studies}

The first author (A.H.) assessed the methodological quality of included studies with reference to the Strengthening the Reporting of Observational Studies in Epidemiology (STROBE) guidelines ${ }^{33}$ and criteria developed by Boyle ${ }^{34}$ to evaluate prevalence studies, which were reviewed and confirmed by the other author (J.P.) as follows:

1. Sample:

a. Explanation of how the sample size was determined;

b. Study population clearly defined;

c. Two-phase sampling process: delirium screening followed by more comprehensive delirium assessment;

d. Minimum of $80 \%$ participation within eligible study population;

2. Measurement:

a. Standardized data collection methods for all participants of the study;

b. Use of valid delirium-screening and assessment tools AND/OR psychiatric assessment;

c. Reporting of measurement reliability processes, for example, user training in the deliriumscreening and assessment tool(s), inter-rater reliability testing, supervision of clinical/research staff conducting study measurements;

3. Analysis:

a. Confidence intervals included for statistical analysis of frequency estimates.

\section{Results}

The initial search generated 815 papers: Scopus $(n=758)$, CINAHL $(n=28)$, Medline $(n=8)$, PubMed via CareSearch $(n=21)$. Within Scopus, adding "AND prospective study," further refined the search and reduced the number of results within Scopus to 84 papers, resulting in 141 papers across all search engines. Once duplicates were removed, 119 papers published between 1980 and 2011 remained (Figure 1). A further 113 papers were removed as they did not report primary research data and/or prospectively measure prevalence or incidence rates of delirium in adult specialist palliative care inpatient units, leaving six papers. Two additional papers ${ }^{35,36}$ were identified from a hand search of the reference lists of the eligible papers and other reviews. ${ }^{1,3}$ At the end of the search, eight studies that prospectively measured the prevalence or incidence of delirium in specialist palliative care adult inpatient settings remained (Table 1). $4,5,35-37,39-41$ These included studies which were conducted in the northern hemisphere over a 12-year period (1996-2008).

\section{Setting, diagnosis, and demographics}

The included studies were undertaken in patient settings described variously as hospices $(n=2),{ }^{39,41}$ palliative care units $(n=3),{ }^{35-37}$ acute palliative care units $(n=2),{ }^{4,40}$ and a combined acute palliative care unit/hospice $(n=1) .{ }^{5}$ Where described, the purpose of the settings included symptom control, respite, rehabilitation, and/or terminal care for palliative care patients. The majority (98.9\%) of all participants $(n=1079)$ across these studies had advanced cancer, with some diagnoses not specified in one study. ${ }^{41}$ Two studies included participants with other life limiting diseases: (a) immunodeficiency disorders $(n=11)^{36}$ and (b) end-stage cardiac failure and cerebrovascular disease $(n=1){ }^{41}$

Across the studies, there was equal representation of males and females, with a mean age of 66.24 years (range 62-68.7 years). Participation rates varied (Table 1).

\section{Study characteristics, design, quality, and focus}

There was variability in study characteristics, design, quality, and foci, as well as participant numbers (X 120, range $41^{40}-228$ people $^{5}$ ). No studies reported statistical explanations for determination of sample size, with this appearing to be largely determined by number of patient admissions within study periods. Delirium occurrence was measured at different frequencies and points of time during the admission, while five studies measured both delirium prevalence and incidence. $4,35,36,39,41$

Different criteria were used to define the terminal stage, with the last weeks of life considered the "pre-terminal and terminal" stage of cancer in two studies. ${ }^{5,39}$ "Terminally ill" or "terminal" cancer patients were elsewhere considered to be within the last 6 months of life. ${ }^{37}$ Only one study included the data specifically collected in the 6 hours immediately prior to death, defined as "terminal delirium." ${ }^{4}$

Methodological quality of studies varied considerably and no study met all quality criteria (Table 1 ).

\section{Definitions of delirium and diagnostic criteria used}

Diagnostic criteria adopted by many of the studies, conducted at different time points, reflect the evolution of the $D S M$ diagnostic criteria for delirium. The majority $(n=6)$ of studies applied DSM criteria to diagnose delirium, with two using the research gold standard of psychiatrist assessment to confirm delirium against the $D S M$ version current at the time. ${ }^{5,37}$ In another four studies, diagnosis of delirium was based on the presence of the then-current DSM criteria, although not confirmed by psychiatric assessment. 4,35,39,41 The remaining two studies used an alternative criteria to establish a delirium diagnosis with one ${ }^{36}$ using the International Statistical Classification of Diseases and Related Health problems (ICD-10) Diagnostic Criteria for Research, ${ }^{42}$ which requires a greater range of symptoms to be present to establish a delirium diagnosis. 


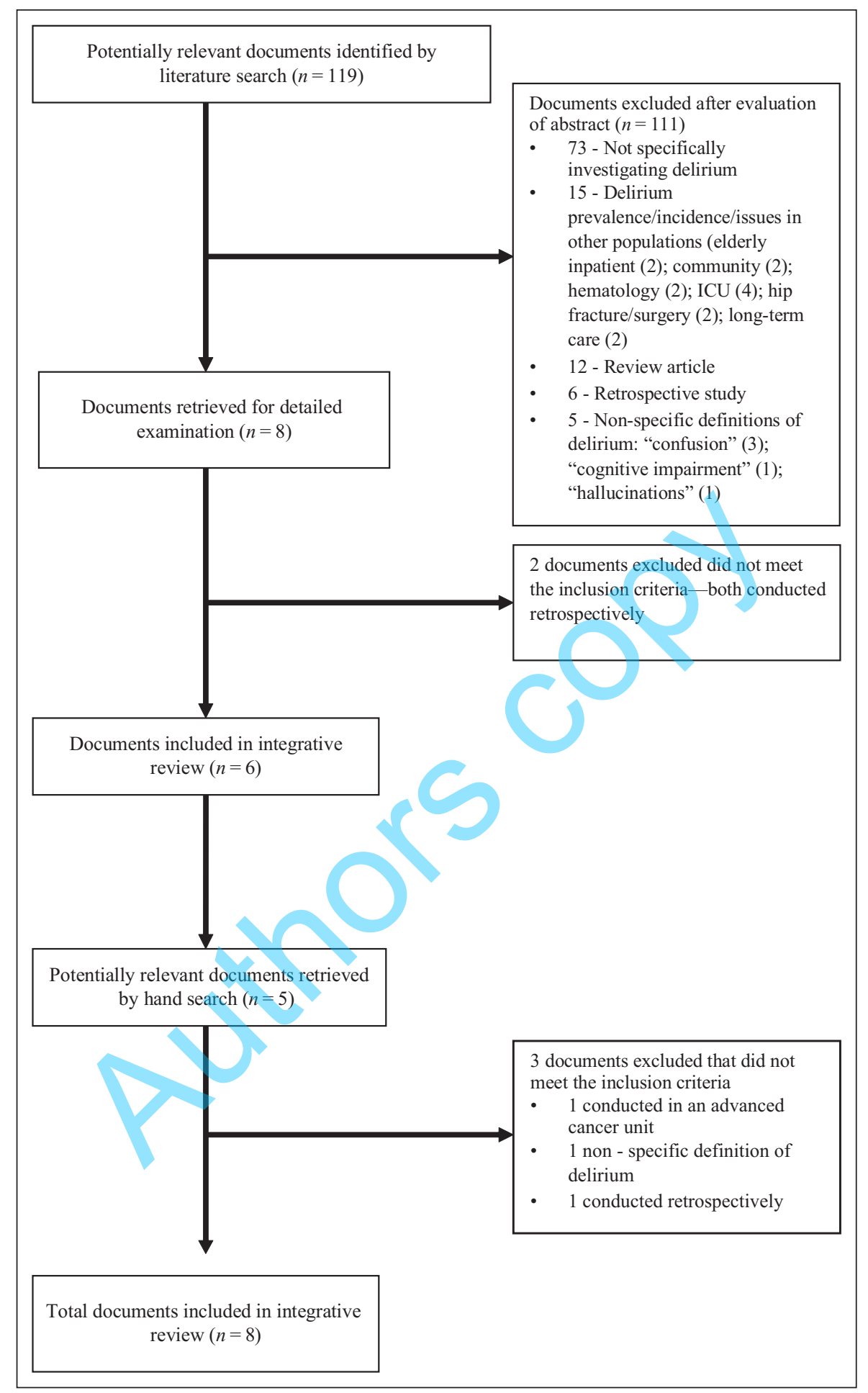

Figure I. Flowchart of studies from search to inclusion.

\section{Screening and assessment tools}

Eight different tools were used across the studies to assess cognition, screen for, or establish delirium (Table 1). Of the six delirium-specific screening or assessment tools, all varied in their validity, purpose (screening, diagnosis, and severity), intended rater (psychiatrically vs nonpsychiatrically trained), ratings procedures (observation vs interview), number of items, and extent to which they correlate with different versions of DSM criteria for delirium. ${ }^{43,44}$

Three delirium or "confusion" screening tools included the Confusion Rating Scale (CRS) used by ward nurses ${ }^{39,45}$; 


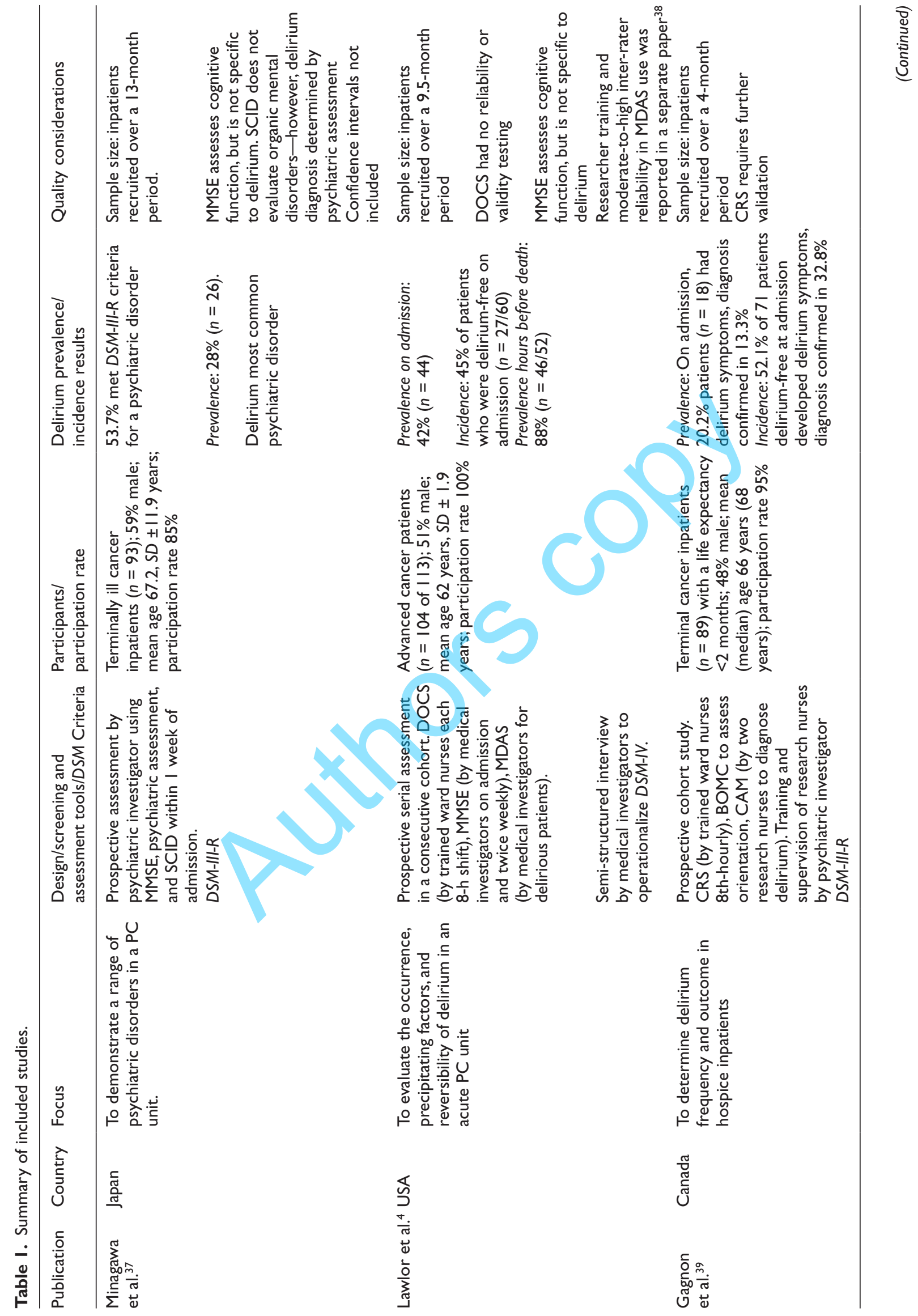




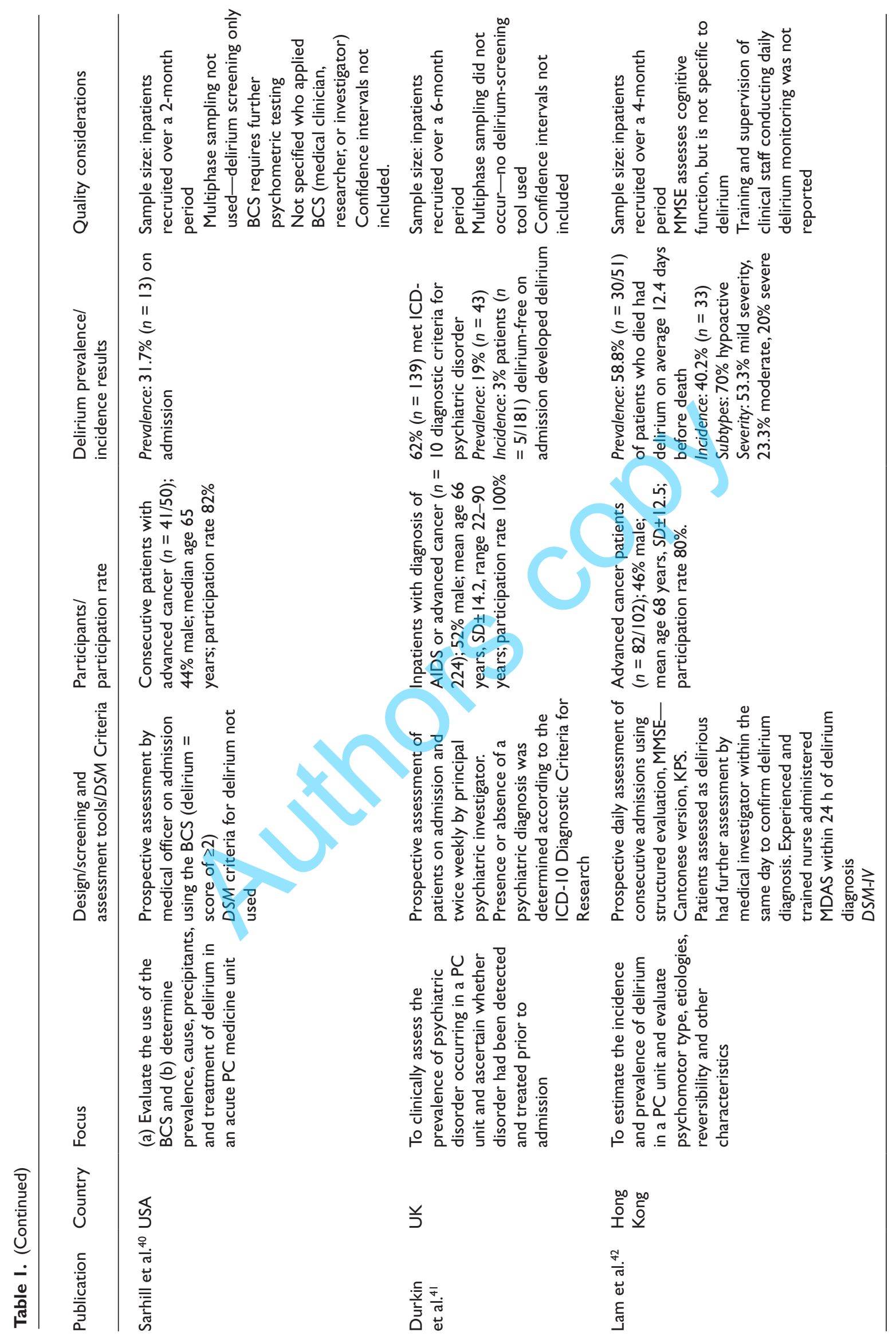







the Bedside Confusion Scale (BCS) used by medical investigators ${ }^{40,46}$; and the Delirium Observational Checklist Scale (DOCS), an instrument developed by study investigators for ward nurse's use. ${ }^{4}$ Although the BCS was previously validated in the palliative care setting, it requires further investigation of its psychometric properties. ${ }^{43}$ The CRS requires further validation, and the DOCS is not a validated delirium-screening tool. ${ }^{43}$

Two cognition assessment tools, used to either screen for delirium or to assist in delirium assessment, were the Mini-Mental State Examination (MMSE) ${ }^{47}$ used by psychiatric and medical investigators $\mathrm{s}^{4,35,37,41}$ or clinical staff ${ }^{41}$ and the Blessed Orientation Memory Concentration (BOMC) test used by research nurses. ${ }^{39,48}$

Three delirium assessment tools were the Confusion Assessment Method (CAM) $)^{49}$ used by research nurses ${ }^{39}$ or medical investigators and trained clinical staff ${ }^{41}$; the Memorial Delirium Assessment Scale (MDAS) ${ }^{38,50}$ used by medical investigators, ${ }^{4,41}$ trained clinical staff, ${ }^{41}$ or a research nurse ${ }^{35}$; and the Delirium Rating Scale-Chinese Version (DRS-CV) ${ }^{51}$ used by a research nurse. ${ }^{5}$ Only the MDAS ${ }^{38,50}$ and the Delirium Rating Scale (DRS) ${ }^{46,52,53}$ were validated in palliative care or advanced cancer populations prior to use in the studies under examination, with the MDAS undergoing further simultaneous validation. ${ }^{4,38,41}$ The $\mathrm{CAM}^{49}$ was validated in other clinical settings and languages, ${ }^{43}$ and subsequently validated in the palliative care setting. ${ }^{54}$

No studies reported perspectives of patients or families of the acceptability of delirium-screening and assessment processes.

\section{Delirium prevalence and incidence rates}

The prevalence and incidence rates reported in the included studies are represented graphically in Figure 2.

On admission. Five studies measured delirium prevalence at admission, ranging from $13.3 \%$ to $42.3 \%$ of patients..$^{4,36,39-41}$ Of 104 advanced cancer admissions to an acute palliative care unit, delirium was present at admission in $42.3 \%$ of patients. ${ }^{4}$ A later study, consecutively measured delirium frequency in hospice inpatients $(n=89)$ and $13.3 \%$ were confirmed to have delirium. ${ }^{39}$ In another, $19 \%$ of patients $(n=224)$ admitted to a palliative care unit had delirium. ${ }^{36} \mathrm{~A}$ third (32\%) of participants $(n=41)$ were classified as delirious according to presence of inattention and altered level of alertness in one acute palliative care unit, ${ }^{40}$ while $29 \%$ of participating patients $(n=100)$ admitted to a Scottish hospice had delirium. ${ }^{41}$

During admission. Delirium prevalence across the whole cohort of palliative care inpatients during each study period ranged from $26 \%$ to $62 \% .5,37,39,41$ One study measuring psychiatric morbidity at one point during the week after

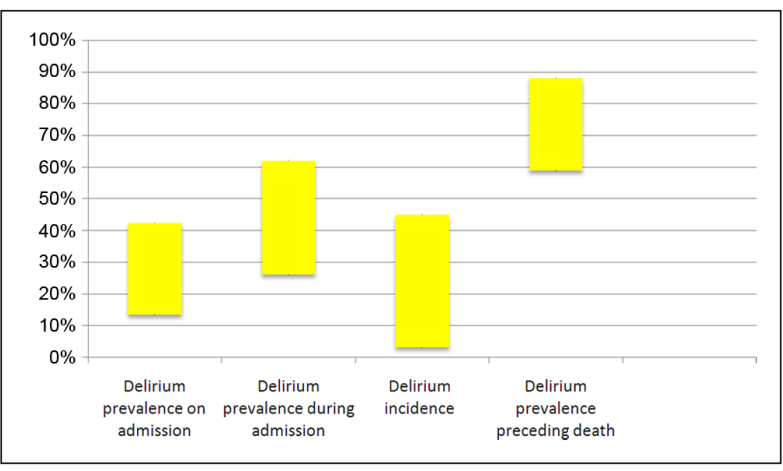

Figure 2. Graphical representation of delirium prevalence and incidence rates in specialist palliative care inpatient units from results of included studies.

admission to a palliative care unit found that delirium was the most prevalent psychiatric disorder, occurring in $28 \%$ of all participants and representing $52 \%$ of all psychiatric diagnoses. ${ }^{37}$ Another study, using delirium screening during each 8 -h shift, identified that $62 \%$ of participants developed delirium at some point during hospice admission. ${ }^{39}$ One study reassessed hospice patients $(n=73) 7$ days after admission and found that $26 \%$ had delirium, while across 8 hospices or inpatient palliative care services, $29.4 \%$ of patients had a delirium diagnosis during a 48-h period of assessment. ${ }^{41}$ Recently, $46.9 \%$ of palliative care inpatients $(n=$ 228) screened second daily were found to have delirium. ${ }^{5}$

Three studies examined occurrence of delirium subtypes and all reported that the majority of delirious patients experienced hypoactive delirium $(68 \%-86 \%))^{5,35,41}$

Five studies measured delirium incidence after admission and reported rates of between $3 \%$ and $45 \%, 4,35,36,39,41$ Delirium developed during admission in $45 \%$ of patients $(n$ $=60) .{ }^{4}$ In a later study involving 71 participants, $32.8 \%$ had confirmed delirium. ${ }^{39}$ These two studies included screening by ward nurses each 8 -h shift. ${ }^{4,39} \mathrm{~A}$ study using daily screening reported, of admitted patients, $40.2 \%(n=82)$ developed delirium $(n=33 / 82)$, the majority $(70 \%)$ having hypoactive delirium of mild severity $(53.3 \%) .{ }^{35}$ In contrast, one study reported development of five new cases in 73 patients within a 7-day period, an incidence of $7 \%,{ }^{41}$ while another, using twice weekly assessment and the ICD-10 diagnostic criteria, reported an incidence of only $3 \%$ : during the 6-month study period only 5 of 181 patients delirium-free on admission subsequently developed delirium. ${ }^{36}$

Preceding death. Two studies measured prevalence of delirium in the weeks or hours before death, reporting rates of $58.8 \%-88 \% .{ }^{4,35}$ The most recent study reported $58 \%$ delirium prevalence in patients $(n=51)$ who died during admission. ${ }^{35}$ Only one study explicitly measured and reported occurrence of delirium in the last $6 \mathrm{~h}$ of life in an 
acute palliative care unit and found that the majority (88\%) had delirium. ${ }^{4}$

\section{Variation in delirium prevalence and incidence according to study methods and settings}

Studies that used $D S M-I V$ criteria reported higher delirium prevalence $(42 \%-88 \%)^{4,5,35}$ and incidence $(40.2 \%-$ $45 \%)^{4,35}$; compared to studies using earlier versions of $D S M$ criteria and ICD-10 (prevalence $13.3 \%-29.4 \%{ }^{36,37,39,41}$ and incidence 3\%-32.8\%). ${ }^{36,39,41}$ Studies screening participants daily or more often reported higher delirium incidence $(32.8 \%-45 \%)^{4,35,39}$ than studies that screened or assessed delirium participants less frequently $(3 \%-$ 7\%). ${ }^{36,41}$ Delirium prevalence on admission varied slightly across settings: palliative care unit (19\%), hospice $(13.3 \%$ $29 \%)$, and acute palliative care units $(31.7 \%-42 \%)$.

\section{Role of clinicians in the identification and diagnosis of delirium}

In four studies, clinicians were actively involved in delirium screening and assessment study processes. ${ }^{4,35,39,41}$ In two, ward nurses screened for delirium, using the $\mathrm{DOCS}^{4}$ or the $\mathrm{CRS}^{39}$ and received training in use of tools and features of delirium. ${ }^{39}$ In another, an experienced and trained nurse assessed delirious patients using the MDAS to measure delirium severity. ${ }^{35}$ In the study involving 8 separate Scottish hospices and palliative care services, clinicians received training prior to using the CAM and MDAS to identify and assess delirium over a 48 -h period. ${ }^{41}$

Research nurses were also involved in delirium screening and assessment. The DRS-CV was used to screen inpatients for delirium, ${ }^{5}$ delirium diagnosis was established by nurses in another using the CAM in consultation with the psychiatric investigator if there was uncertainty about the diagnosis, ${ }^{39}$ and delirium severity was measured by nurses using the MDAS $24 \mathrm{~h}$ after delirium diagnosis by a physician. ${ }^{35}$

\section{Discussion}

There were some similarities across studies, with most adopting a two-phase sampling method-delirium screening followed by assessment - and involving patients of a similar age and primary cancer diagnosis. However, there was a varying methodological quality across these studies, with heterogeneity of diagnostic criteria, sample sizes, frequency of assessment, and measurement tools adopted. Despite these differences and the variation in reported delirium occurrence, categorizing delirium prevalence at different points along the palliative care inpatient trajectory indicates that prevalence is lower at admission (range $13.3 \%-42.3 \%$ ) ${ }^{4,36,39-41}$ increases during admission (range
$26 \%-62 \%), 5,37,39,41$ with the risk of developing delirium escalating as death nears (range $58.8 \%-88 \%$ ). ${ }^{4,35}$ This review has confirmed that palliative care inpatient populations have delirium incidence and prevalence equal to or greater than other known high-risk populations, such as older people admitted to hospital, ${ }^{55}$ Intensive Care Units, ${ }^{56-58}$ post hip surgery, ${ }^{59,60}$ and long-term care. ${ }^{61}$

The review adds to the emerging evidence that hypoactive delirium is the most prevalent subtype in palliative care populations. $5,35,41,62$ Hypoactive delirium may appear less severe than other subtypes ${ }^{35}$ and cause less difficulties in ward management, ${ }^{63}$ but is associated with increased mortality. ${ }^{5}$ It also has a significant impact on patients and families since cognitive changes occur as often as in the hyperactive and mixed subtypes. ${ }^{62,64}$

Clinicians were involved in patient screening and assessment in half the studies, highlighting potential for routine delirium screening outside a research context, and feasibility of increasing delirium recognition capabilities by nonpsychiatric clinicians through training and access to validated delirium-screening and assessment tools. ${ }^{38,50,54}$ Delirium screening by nurses in a hospice setting has been demonstrated to be feasible and effective..$^{14,25}$ However, the challenges of screening for delirium in palliative care populations was also demonstrated by the small proportion of included studies measuring delirium occurrence specifically in cohorts of patients who were dying, and proportion of patients and/or families who declined to participate in the delirium assessment process, indicating delirium assessment is not always acceptable to them. Additionally, many patients were too unwell to provide consent or were excluded because they were dying, comatose, or could not speak. Similarly, a recent study ${ }^{14}$ reported a low rate of CAM completion by hospice nurses (39\%), highlighting the difficulty of conducting this delirium assessment in the last days of life and need for validated low-burden delirium assessment tools at this time.

Applying the DSM-IV criteria appears to lead to increased case finding, which has been previously reported ${ }^{65}$; and interestingly, variability in delirium prevalence and incidence noted in this review reflects results of similar reviews that included studies using less-specific delirium definitions. ${ }^{1,3}$

\section{Implications for clinical practice and future research}

As daily screening increases detection of incident delirium, $, 435,39$ the question remains: should routine screening be implemented in palliative care inpatient settings? Clinical practice guidelines for other high-risk patient populations recommend screening to improve early recognition of delirium, ${ }^{55,66,67}$ although the extent to which this has been routinely adopted is unknown. However, a number of other key questions require investigation to justify routine screening 28,29 including: Is screening acceptable to patients and 
family and cause minimal harm? Is it cost-effective? Does early recognition and treatment of delirium improve mortality and morbidity? And, what are the adverse effects of delirium treatment? ${ }^{28,29}$

Further research in delirium prevention interventions in palliative care, ${ }^{14}$ and high-level evidence of the efficacy and safety of pharmacological interventions, such as antipsychotics, benzodiazepines, and methylphenidate, is needed. ${ }^{16,68,69}$ Measuring impact of interventions on delirium incidence, severity, and patient mortality should continue to be a focus of research, but as improvements in morbidity and mortality are likely to be minimal in this population and the focus of care is a relief of distress and suffering, patients' and families' subjective experiences (such as perceptions of care, distress, dignity, and quality of life) related to delirium screening, recognition, and treatment are especially important outcomes to be determined. ${ }^{70}$ Development of acceptable, observational delirium screening and assessment strategies for palliative care patients who are very ill, dying, or unable to communicate is also required. ${ }^{8,28,29}$

This review has highlighted the lack of consensus regarding selection of delirium screening and assessment tools in palliative care research, and this is likely to be reflected in clinical practice. Establishing the acceptability of various delirium-screening and assessment tools by patients and families would inform the sector about which are the most appropriate to use in this population, particularly in the dying stage. Establishing consensus would facilitate delirium benchmarking, quality improvement, ${ }^{71,72}$ and consistency of research methodology. To further improve methodological and reporting quality of future delirium epidemiological research in palliative care populations, consideration of recently developed guidelines for observational studies in epidemiology is recommended. ${ }^{33,73}$

Health-economic analysis will also be an important inclusion in future delirium research, when high healthcare costs associated with delirium occurrence in elderly inpatient populations is considered. ${ }^{74,75}$

\section{Study limitations and strengths}

Limitations of this review include exclusion of papers not published in English, potentially contributing to selection bias and the absence of multiple independent raters in the extraction of data to assess eligibility and quality of included studies. There are limitations related to generalizability of this review due to the focus on advanced cancer diagnoses within study populations. ${ }^{70}$ As the brief of palliative care shifts to nonmalignant conditions and settings where end-of-life care is routinely provided, for example, elderly medical inpatient settings or nursing homes, it is important to consider implications of this changing population. ${ }^{76-78}$ In addition, although results suggest increasing delirium prevalence as death nears, this was not confirmed within this review due to variable reporting of participants' functional status and illness staging, and variation in operational definitions of "terminal." This barrier has been previously noted with a recommendation that all future delirium occurrence studies incorporate a patient cohort classification system based on estimated prognosis. ${ }^{3}$

The strengths of this review include use of a systematic approach, with application of accepted guidelines and a structured approach to the assessment of quality of included studies. ${ }^{30,33}$

\section{Conclusion}

This review has examined methods, quality, and results of studies prospectively measuring delirium occurrence in specialist palliative care inpatient settings and identified additional evidence needed to justify routine delirium screening in these settings. While the moderate to high rate of delirium occurrence in palliative care inpatient units supports the need for delirium screening, there is also a need to develop consensus and quality of methods for measuring delirium occurrence, and we require evidence regarding impact, acceptability, potential harms and cost-effectiveness of delirium screening and assessment, and outcomes of screening and treatments on morbidity, mortality, and patients' and families' subjective experiences.

\section{Acknowledgements}

The authors would like to acknowledge the contribution of Ms Caroline Yeh, Librarian, St Vincent's Hospital, Darlinghurst, Sydney, Australia in the development of the literature search strategy.

\section{Funding}

An Australian Postgraduate Award from the Commonwealth Government of Australia supported A.H. in this work.

\section{References}

1. Leonard M, Agar M, Mason C, et al. Delirium issues in palliative care settings. J Psychosom Res 2008; 65(3): 289-298.

2. Friedlander MM, Yanina B and Breitbart WS. Delirium in palliative care. Oncology 2004; 18(12): 1541-1550.

3. Hjermstad MJ, Loge JH and Kaasa S. Methods for assessment of cognitive failure and delirium in palliative care patients: implications for practice and research. Palliat Med 2004; 18(6): 494-506.

4. Lawlor PG, Gagnon B, Mancini IL, et al. Occurrence, causes, and outcome of delirium in patients with advanced cancer: a prospective study. Arch Intern Med 2000; 160(6): 786-794.

5. Fang CK, Chen HW, Liu SI, et al. Prevalence, detection and treatment of delirium in terminal cancer inpatients: a prospective survey. Jpn J Clin Oncol 2008; 38(1): 56-63. 
6. Breitbart W, Gibson C and Tremblay A. The delirium experience: delirium recall and delirium-related distress in hospitalized patients with cancer, their spouses/caregivers, and their nurses. Psychosomatics 2002; 43(3): 183-194.

7. Bruera E, Bush SH, Willey J, et al. Impact of delirium and recall on the level of distress in patients with advanced cancer and their family caregivers. Cancer 2009; 115(9): 2004-2012.

8. O'Malley G, Leonard M, Meagher D, et al. The delirium experience: a review. J Psychosom Res 2008; 65(3): 223-228.

9. Brajtman $\mathrm{S}$. The impact on the family of terminal restlessness and its management. Palliat Med 2003; 17(5): 454-460.

10. Namba M, Morita T, Imura C, et al. Terminal delirium: families' experience. Palliat Med 2007; 21(7): 587-594.

11. Morita T, Akechi T, Ikenaga M, et al. Terminal delirium: recommendations from bereaved families' experiences. J Pain Symptom Manage 2007; 34(6): 579-589.

12. American Psychiatric Association. DSM-IV-TR: diagnostic and statistical manual of mental disorders. 4th ed. text rev. Washington, DC: American Psychiatric Association, 2000.

13. De Rooij SE, Schuurmans MJ, Van Der Mast RC, et al. Clinical subtypes of delirium and their relevance for daily clinical practice: a systematic review. Int J Geriatr Psychiatry 2005; 20(7): 609-615.

14. Gagnon P, Allard P, Gagnon B, et al. Delirium prevention in terminal cancer: assessment of a multicomponent intervention. Psychooncology 2012; 21(2): 187-194.

15. Jackson KC and Lipman AG. Drug therapy for delirium in terminally ill adult patients. Cochrane DB Syst Rev [serial on the Internet] 2004; Issue no. 2, http://www.mrw.interscience. wiley.com/cochrane/clsysrev/articles/CD004770/frame.html

16. Elie D, Gagnon P, Gagnon B, et al. Using psychostimulants in end-of-life patients with hypoactive delirium and cognitive disorders: a literature review. Can J Psychiatry 2010; 55(6): 386-393.

17. Leonard M, Raju B, Conroy M, et al. Reversibility of delirium in terminally ill patients and predictors of mortality. Palliat Med 2008; 22(7): 848-854.

18. Gaudreau J, Gagnon P, Roy M, et al. Opioid medications and longitudinal risk of delirium in hospitalized cancer patients. Cancer 2007; 109(11): 2365-2373.

19. Gaudreau J, Gagnon P, Harel F, et al. Psychoactive medications and risk of delirium in hospitalized cancer patients. J Clin Oncol 2005; 23(27): 6712-6718.

20. Cowan JD and Walsh D. Terminal sedation in palliative medicine definition and review of the literature. Support Care Cancer 2001; 9(6): 403-407.

21. Fainsinger RL, De Moissac D, Mancini I, et al. Sedation for delirium and other symptoms in terminally ill patients in Edmonton. J Palliat Care 2000; 16(2): 5-10.

22. Barnes J, Kite S and Kumar M. The recognition and documentation of delirium in hospital palliative care inpatients. Palliat Support Care 2010; 8(2): 133-136.

23. Irwin SA, Rao S, Bower KA, et al. Psychiatric issues in palliative care: recognition of delirium in patients enrolled in hospice care. Palliat Support Care 2008; 6(2): 159-164.
24. Agar M, Draper B, Phillips PA, et al. Making decisions about delirium: a qualitative comparison of decision making between nurses working in palliative care, aged care, aged care psychiatry, and oncology. Palliat Med 2011; Epub ahead of print September 9, 2011, DOI: 10.1177/0269216311419884.

25. Rao S, Ferris FD and Irwin SA. Ease of screening for depression and delirium in patients enrolled in inpatient hospice care. J Palliat Med 2011; 14(3): 275-279.

26. Smith J and Adcock L. The recognition of delirium in hospice inpatient units. Palliat Med 2012; 26(3): 283-285.

27. Breitbart W and Alici Y. Agitation and delirium at the end of life: "We couldn't manage him." JAMA 2008; 300(24): 2898-2910.

28. Harris RP, Helfand M, Woolf SH, et al. for the Methods Word Group, third U.S. Preventive Services Task Force. Current methods of the U.S. preventive services task force: a review of the process. Am J Prev Med 2001; 20(3S): 21-35.

29. Wilson JMG and Jungner G. Principles and practice of screening for disease Geneva. Available at: http://www.who. int/bulletin/volumes/86/4/07-050112BP.pdf. Public Health Papers, \#34, 1968. Geneva: World Health Organization.

30. Stroup DF, Berlin JA, Morton SC, et al. Meta-analysis of observational studies in epidemiology - a proposal for reporting. JAMA 2000; 283(15): 2008-2012.

31. American Psychiatric Association (ed.). DSM-III diagnostic and statistical manual of mental disorders. 3rd ed. Washington, DC: American Psychiatric Association, 1980.

32. Tieman J, Sladek R, and Currow D, Multiple sources: mapping the literature of palliative care. Palliat Med, 2009. 23(5): 425-431.

33. Von Elm E, Altman DG, Egger M, et al. The Strengthening the Reporting of Observational Studies in Epidemiology (STROBE) statement: guidelines for reporting observational studies. PLoS Med 2007; 4(10): e296.

34. Boyle MH. Guidelines for evaluating prevalence studies. Evid Base Ment Health 1998; 1(2): 37-39.

35. Lam PT, Tse CY and Lee CH. Delirium in a palliative care unit. Prog Palliat Care 2003; 13(3): 126-133.

36. Durkin I, Kearney M and O'Siorain L. Psychiatric disorder in a palliative care unit. Palliat Med 2003; 17(2): 212-218.

37. Minagawa H, Uchitomi Y, Yamawaki S, et al. Psychiatric morbidity in terminally ill cancer patients: a prospective study. Cancer 1996; 78(5): 1131-1137.

38. Lawlor PG, Nekolaichuk C, Gagnon B, et al. Clinical utility, factor analysis, and further validation of the memorial delirium assessment scale in patients with advanced cancer. Cancer 2000; 88(12): 2859-2867.

39. Gagnon P, Allard P, Mâsse B, et al. Delirium in terminal cancer: a prospective study using daily screening, early diagnosis, and continuous monitoring. J Pain Symptom Manage 2000; 19: 412-426.

40. Sarhill N, Walsh D, Nelson KA, et al. Assessment of delirium in advanced cancer: the use of the Bedside Confusion Scale. Am J Hosp Palliat Care 2001; 18(5): 335-341.

41. Spiller JA and Keen JC. Hypoactive delirium: assessing the extent of the problem for inpatient specialist palliative care. Palliat Med 2006; 20(1): 17-23. 
42. World Health Organization. The ICD-10 classification of mental and behavioural disorders. Diagnostic criteria for research. Geneva: World Health Organization, 1993.

43. Adamis D, Sharma N, Whelan P, et al. Delirium scales: a review of current evidence. Aging Ment Health 2010; 14(5): 543-555.

44. Smith MJ, Breitbart WS and Platt MM. A critique of instruments and methods to detect, diagnose, and rate delirium. $J$ Pain Symptom Manage 1995; 10(1): 35-77.

45. Williams MA. Delirium/acute confusional states: evaluation devices in nursing. Int Psychogeriatr 1991; 3(02): 301-308.

46. Stillman MJ and Rybicki LA. The Bedside Confusion Scale: development of a portable bedside test for confusion and its application to the palliative medicine population. $J$ Palliat Med 2000; 3(4): 449-456.

47. Folstein MF, Folstein SE and McHugh PR. "Mini-Mental State." A practical method for grading the cognitive state of patients for the clinician. J Psychiatr Res 1975; 12: 189-198.

48. Katzman R, Brown T and Fuld P. Validation of a short orientation-memory-concentration test of congestive impairment. Am J Psychiatry 1983; 140(6): 734-739.

49. Inouye SK, Van Dyck CH, Alessi CA, et al. Clarifying confusion: the confusion assessment method. A new method for detection of delirium. Ann Intern Med 1990; 113(12): 941-948.

50. Breitbart W, Rosenfeld B, Roth A, et al. The memorial delirium assessment scale. J Pain Symptom Manage 1997; 13(3): 128-137.

51. Trzepacz PT, Baker RW and Greenhouse J. A symptom rating scale for delirium. Psychiatr Res 1988; 23: 89-97.

52. Trzepacz PTR, Kanary K, Norton J, et al. Validation of the Delirium Rating Scale-Revised-98: comparison with the Delirium Rating Scale and the Cognitive Test for Delirium. $J$ Neuropsychiatry Clin Neurosci 2001; 13(2): 229-242.

53. Grassi L, Caraceni A, Beltrami E, et al. Assessing delirium in cancer patients. The Italian versions of the Delirium Rating Scale and the Memorial Delirium Assessment Scale. J Pain Symptom Manage 2001; 21(1): 59-68.

54. Ryan K, Leonard M, Guerin S, et al. Validation of the confusion assessment method in the palliative care setting. Palliat Med 2009; 23: 40-45.

55. Clinical Epidemiology and Health Service Evaluation Unit MH. Clinical practice guidelines for the management of delirium in older people. 2006. "Australian Health Ministers' Advisory Council (AHMAC): Melbourne.

56. Ely EW, Inouye SK, Bernard GR, et al. Delirium in mechanically ventilated patients: validity and reliability of the confusion assessment method for the intensive care unit (CAM-ICU). JAMA 2001; 286(21): 2703-2710.

57. Ely EW, Margolin R, Francis J, et al. Evaluation of delirium in critically ill patients: validation of the Confusion Assessment Method for the Intensive Care Unit (CAM-ICU). Crit Care Med 2001; 29(7): 1370-1379.
58. McNicoll L, Pisani MA, Ely EW, et al. Detection of delirium in the intensive care unit: comparison of confusion assessment method for the intensive care unit with confusion assessment method ratings. J Am Geriatr Soc 2005; 53(3): 495-500.

59. Galanakis P, Bickel H, Gradinger R, et al. Acute confusional state in the elderly following hip surgery: incidence, risk factors and complications. Int J Geriatr Psychiatry 2001; 16(4): 349-355.

60. Santana Santos F, Wahlund LO, Varli F, et al. Incidence, clinical features and subtypes of delirium in elderly patients treated for hip fractures. Dement Geriatr Cogn Disord 2005; 20(4): 231-237.

61. McCusker J, Cole MG, Voyer P, et al. Prevalence and incidence of delirium in long-term care. Int J Geriatr Psychiatry 2011; 26: 1152-1161.

62. Leonard M, Donnelly S, Conroy M, et al. Phenomenological and neuropsychological profile across motor variants of delirium in a palliative-care unit. J Neuropsychiatry Clin Neurosci 2011; 23(2): 180-188

63. Meagher DJ, Leonard M, Donnelly S, et al. A longitudinal study of motor subtypes in delirium: relationship with other phenomenology, etiology, medication exposure and prognosis. J Psychosom Res 2011; 71(6): 395-403.

64. Steinhauser KE, Christakis NA, Clipp EC, et al. Factors considered important at the end of life by patients, family, physicians, and other care providers. JAMA 2000; 284(19): 2476-2482.

65. Cole MG, Dendukuri N, McCusker J, et al. An empirical study of different diagnostic criteria for delirium among elderly medical inpatients. J Neuropsychiatry Clin Neurosci 2003; 15(2): 200-207.

66. Canadian Coalition for Seniors' Mental Health (CCSMH). Guideline on the assessment and treatment of delirium in older adults at the end of life. 2010. Canadian Coalition for Seniors' Mental Health ( CCSMH ): Toronto.

67. National Clinical Guideline Centre for Acute and Chronic Conditions, Delirium: diagnosis, prevention and management, NICE Clinical Guideline 103, 2010, National Institute for Health and Clinical Excellence: London.

68. Agar M. Randomised control trial of oral risperidone, oral haloperidol, and oral placebo with rescue subcutaneous midazolam in the management of delirium in palliative care inpatients [Clinical Trial Protocol]. Adelaide, SA, Australia: Flinders University, 2010; http://www.caresearch. com.au/Caresearch/Portals/0/Documents/RESEARCHRESOURCES/Research-Studies-Register/0071.pdf

69. Lonergan E, Luxenberg J and Areosa Sastre A. Benzodiazepines for delirium. Cochrane DB Syst Rev [serial on the Internet] 2009; Issue no. 4, http://www.mrw.interscience. wiley.com/cochrane/clsysrev/articles/CD006379/frame.html

70. World Health Organization. WHO Definition of Palliative Care. 2002; http://www.who.int/cancer/palliative/definition/en/

71. Eagar K. The Australian Palliative Care Outcomes Collaboration (PCOC) - measuring the quality and outcomes of 
palliative care on a routine basis. Aust Health Rev 2010; 34(2): 186-192.

72. Cicely Saunders Institute. Palliative Care Outcome Scale. London. http://pos-pal.org/index.php (accessed 29 March 2012).

73. Vandenbroucke JP, Von Elm E, Altman DG, et al. Strengthening the Reporting of Observational Studies in Epidemiology (STROBE): explanation and elaboration PLoS Med 2007; 4(10), e297. PMID: 17941715.

74. Leslie DL, Zhang Y, Bogardus ST, et al. Consequences of preventing delirium in hospitalized older adults on nursing home costs. J Am Geriatr Soc 2005; 53(3): 405-409.
75. Leslie DL, Marcantonio ER, Zhang Y, et al. One-year health care costs associated with delirium in the elderly population. Arch Intern Med 2008; 168(1): 27-32.

76. Murray SK, Kendall M, Boyd K, et al. Illness trajectories and palliative care. BMJ 2005; 330: 1007-1011.

77. World Health Organisation. Palliative care for older people: better practices. Sue Hall, Hristina Petkova, Agis D. Tsouros, Massimo Costantini and Irene J. Higginson, Eds, 2011, WHO Regional Office for Europe: Copenhagen.

78. Janssen DJA, Spruit MA, Wouters EFM, et al. Daily symptom burden in end-stage chronic organ failure: a systematic review. Palliat Med 2008; 22(8): 938-948. 